\title{
Review of: "Association of visual impairment with cognitive decline among older adults in Taiwan"
}

\author{
William Monaco
}

Potential competing interests: The author(s) declared that no potential competing interests exist.

The article is well written, timely and comprehensive. This retrospective, large-population, cohort study explored extensive clinical data, including visual acuity and cognitive measures, as well as systemic, social and demographic data were collected from a population of 105, 208 ambulatory Taipei and Aboriginal citizens in Taiwan over a period of 7 years. Their age was 65 and older. The research was well designed and focused on two specific aims: The association between vision loss and cognitive decline in a population of Asian elderly, and to determine other potential risk factors and their combined and independent influence with vision loss on the development and progression of cognitive decline. The authors identified some limitations in the study, including their use of general population screening tools for cognitive testing, as well as some restrictions in how the visual acuity was measured and recorded. There was a minor inconsistency in reporting the subject's age as it is reported in the methods it is stated as greater than 65 and in the Introduction, Abstract and text it is stated as age 65 and greater. However, their results are compelling and address a key questions that have been raised by key aging investigators and research organizations in the U.S. (Lancet Global Health 2021, online 2-16-21, Burton, M et. al. : J. Am. Geriatr. Soc. 3-18, Whitson, H et. al.).

Vision and cognitive data were recorded by the best means available and the resultant summary data provide reasonable documentation for the evidence that, in subjects experiencing vision loss, there was cumulative cognitive decline, particularly in the blind population, and the hazard ratio for potential cognitive decline was 2 and 5 times higher for vision impaired and blind, respectively in each of the major clinical subgroups.

These data provide substantial supporting evidence for vision loss independently contributing to incident cognitive decline and refuting earlier suggestions that there was no significant relationship between vision loss and cognitive decline. Further, this work further substantiates the need for regular, comprehensive, eye care exams for the elderly, who are at higher risk of age related eye disease, particularly those who are institutionalized and may not have access to the care (JAMDA, 2021, Monaco, W et. al). 